\title{
Blood pressure management during beach chair position shoulder surgery: What do we know?
}

\author{
Glenn S. Murphy, MD · Joseph W. Szokol, MD
}

Received: 12 July 2011 / Accepted: 10 August 2011 / Published online: 24 August 2011

(C) Canadian Anesthesiologists' Society 2011

One of the surgical procedures most commonly performed in North America is arthroscopic repair of the shoulder joint. In the United States, approximately two-thirds of these patients undergo anesthesia and surgery in the sitting or beach chair position (BCP). ${ }^{1}$ Although the BCP offers several advantages for the surgeon, including reduced risk of neurovascular trauma, ease of conversion to an open approach, and excellent intra-articular visualization, ${ }^{2}$ the sitting position introduces several challenges for the anesthesiologist and potential risks to the patient. Recent reports have described catastrophic neurologic injuries occurring in otherwise healthy patients during shoulder surgery in the sitting position. ${ }^{3-5}$ The authors of these reports hypothesized that improper blood pressure management resulted in reductions in cerebral perfusion pressure which led to central nervous system ischemia and injury. At the present time, however, several important questions about "best practices" for blood pressure management in the $\mathrm{BCP}$ remain unanswered. In this issue of the Journal, the investigations by YaDeau et al. ${ }^{6}$ and Trentman et $a{ }^{7}{ }^{7}$ provide important data in this ongoing debate about the prevalence, risk factors, and potential consequences of hypotension during shoulder surgery in the sitting position.

The incidence of ischemic brain or spinal cord injury following shoulder surgery in the BCP is unknown. In 2003, Bhatti and Enneking reported that a patient developed visual loss and ophthalmoplegia after shoulder surgery. ${ }^{4}$ In 2005, Pohl and Cullen described four cases of severe brain and spinal cord injury in this patient

G. S. Murphy, MD (凹) · J. W. Szokol, MD

Department of Anesthesiology, NorthShore University

HealthSystem, University of Chicago Pritzker School of

Medicine, 2650 Ridge Avenue, Evanston, IL 60201, USA

e-mail: dgmurphy2@yahoo.com population. ${ }^{3}$ In a 2009 survey of 287 members of the American Shoulder and Elbow Surgeons Society (93 responses), eight cases of cerebral vascular events after shoulder surgery were reported, all of which occurred in the sitting position. ${ }^{1}$ An analysis of the American Society of Anesthesiologists Closed Claims database for new onset cervical cord injuries published this year revealed that $24 \%$ of the events occurred in patients having surgery in the sitting position. ${ }^{5}$ It is likely that the incidence of these complications is significantly underreported, as surgeons and anesthesiologists may be reluctant to publish cases where otherwise healthy patients suffered severe neurologic injuries. Through personal communications, the authors of this editorial are aware of eight additional cases of central nervous system injury following BCP surgery that have not been reported in the literature. The Anesthesia Patient Safety Foundation (APSF) has now funded a national registry, the Neurologic Injury after Non-Supine Shoulder Surgery (NINSS) registry (http://depts.washington.edu/asaccp/NINS/ index.shtml), to establish the incidence of central nervous system injury and determine probable causative factors.

The adverse neurologic events reported thus far were attributed most commonly to inadequate cerebral perfusion secondary to intraoperative hypotension. Significant hemodynamic changes occur when the patient's position is changed from supine to sitting. In awake subjects, upright positioning activates the sympathetic nervous system, producing increases in systemic vascular resistance (30$40 \%$ ) and systemic blood pressure (10-15\%) and reductions in cardiac output (15-20\%). ${ }^{8}$ Under general anesthesia, baroreceptor responses are blunted, resulting in an attenuated increase in systemic vascular resistance, a decrease in mean arterial pressure, and a greater reduction in cardiac output compared with the awake state. ${ }^{9}$ Not surprisingly, 
because of these pronounced physiologic changes, a high incidence of intraoperative hypotension (12-32\%) has been observed in patients undergoing surgery under general anesthesia in the sitting position. ${ }^{9}$

At the present time, two critical questions about blood pressure management in the $\mathrm{BCP}$ are actively being debated. The first question is related to the safe lower limit of autoregulation of the brain. Traditionally, the "acceptable" lower limit of autoregulation was thought to be a mean arterial pressure of approximately $50 \mathrm{mmHg}$. However, a number of investigations published in the last six decades have demonstrated that this threshold may be as high as $60-90 \mathrm{mmHg}$ in awake normotensive volunteers. ${ }^{10}$ As stated in a recent review, available evidence supports a mean arterial pressure of $70 \mathrm{mmHg}$ as the lower limit of autoregulation of the brain in supine non-anesthetized subjects. ${ }^{10}$ These data suggest that the relatively common practice of induced hypotension under anesthesia may compromise cerebral perfusion in some at-risk patients, particularly hypertensive individuals whose lower limit of autoregulation might be shifted to the right. Unfortunately, there is no non-invasive technique to determine the lower limit of autoregulation in an individual patient (although preliminary data suggest that cerebral oximetry may be useful in determining this endpoint). The second important question concerns whether blood pressure should be corrected to account for the difference in height between the site of measurement (most commonly a non-invasive cuff on the non-operative arm) and the brain. There are two theories currently being debated. The siphon concept, or "closed model", postulates that a continuous column of blood exists between the arterial and venous limbs of the cerebral circulation. ${ }^{\text {A }}$ Since gravitational effects are identical on the ascending and descending limbs of the loop, no "correction factor" is needed; the sitting position results in equivalent decreases in mean arterial pressure and central venous pressure, resulting in no change in cerebral perfusion pressure. According to the waterfall concept, or "open model", collapsible veins prevent a siphon effect from operating. ${ }^{A}$ Gravitational effects cause blood to "fall" on the venous side of the cerebral circuit, and the heart is responsible for pumping a column of blood towards the brain. Therefore, a correction factor is needed to account for the weight of this column of blood (a reduction in blood pressure of $1 \mathrm{mmHg}$ for each $1.25 \mathrm{~cm}$ height difference between the site of measurement and the brain). There is no consensus at this time about whether blood pressure should be "corrected" in the BCP. Even so, many (or most)

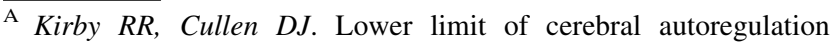
questioned. APSF Newsletter 2009; 24: 5.
}

neuroanesthesiologists manage blood pressure according to the waterfall concept when sitting position surgery is used and thus raise the arterial transducer to the level of the Circle of Willis. It is likely that most anesthesiologists do not use a similar blood pressure correction factor during shoulder surgery in the BCP.

The study by YaDeau et al. was designed to examine the effect of controlled hypotension on cerebral oxygenation in patients undergoing BCP shoulder arthroscopy with regional anesthesia. ${ }^{6}$ Cerebral oximetry was used to assess the adequacy of brain oxygenation and, indirectly, that of cerebral blood flow. Although neurologic injuries are relatively rare after BCP surgery, major cerebral desaturation events (CDE) - defined as a decrease in cerebral oxygen saturation ( $\mathrm{rSO} 2$ ) of $\geq 20 \%$ from baseline-are more common during shoulder arthroscopy and may serve as "early warning signs" of brain malperfusion, ischemia, and infarction. ${ }^{11}$ The study was performed at an institution where controlled hypotension is commonly used in orthopedic patients. Hypotension was classified using preestablished definitions, and blood pressure was corrected to account for the distance between the heart and the head (waterfall concept). There are several limitations to the study design, e.g., type and depth of sedation were not standardized (level of sedation may influence $\mathrm{rSO} 2$ values); no general anesthesia group was included as a comparison cohort; no standard definition of "controlled hypotension" was utilized; a variety of agents were administered to induce hypotension, which may alter cerebral vasoreactivity and $\mathrm{rSO} 2$ values via different mechanisms; and epinephrine was used in the regional blocks, which may have an impact on $\mathrm{rSO} 2$ values by increasing cardiac output and cerebral blood flow during hypotensive episodes. Despite these limitations, there are two important findings from this investigation. First, CDE were uncommon and their duration brief when regional anesthesia was used. In contrast, $80 \%$ of patients have major CDE when BCP surgery is performed under general anesthesia with controlled ventilation. ${ }^{11}$ These observations suggest that cerebral blood flow is better maintained in the BCP when regional anesthesia is used. The reduced incidence of $\mathrm{CDE}$ in patients receiving interscalene or supraclavicular nerve blocks may be due to better preservation of hemodynamic indices under regional anesthesia (see above) or related to higher arterial carbon dioxide values (and associated improvements in cerebral blood flow), which are typically observed in the sedated spontaneously breathing patient as opposed to the patient receiving controlled ventilation and general anesthesia. Second, no clear association between hypotension and CDE was observed. The relationship between blood pressure and CDE has not been examined when BCP surgery is performed under general anesthesia. Although the present 
study provides support for the safety of controlled hypotension when regional anesthesia is used, further data are needed before this practice can be recommended. It is possible that intraoperative hypotension may result in more subtle neurocognitive dysfunction and cerebral injury which are not detected easily on routine clinical exams.

Cerebral desaturation events are common in BCP surgery under general anesthesia, even when blood pressure is maintained within $20 \%$ of baseline values. ${ }^{11}$ Hypotension is likely less well tolerated in this patient population. In the investigation by Trentman et al., they examined the incidence of hypotension in patients undergoing general anesthesia during shoulder surgery in the BCP. ${ }^{7}$ Although the use of antihypertensive agents has been shown to be associated with a greater incidence of hypotension in supine patients under general anesthesia, ${ }^{12}$ this relationship has not been previously examined in sitting patients. Medical records were reviewed retrospectively to determine the incidence of hypotensive episodes (systolic blood pressure $\leq 85 \mathrm{mmHg}$ - not corrected to account for differences in height between site of measurement and the brain) in normotensive or treated hypertensive patients. Even after adjusting for risk factors, such as age and body mass index, the investigators observed that the incidence of hypotension was significantly higher in patients receiving preoperative antihypertensive medications. Although subject to limitations inherent in a retrospective investigation (i.e., no standardization of anesthetic, surgical, or blood pressure management), this study is important because it demonstrated that patients treated with preoperative antihypertensive agents are at risk for hypotension when the BCP is used with general anesthesia. The risk of hypotension is even greater if blood pressure is assessed and corrected using the waterfall concept.

What is the range of "ideal systemic target pressures" that can be maintained in the $\mathrm{BCP}$ without producing neurologic injury? There is insufficient data at the present time to answer this important question. At the 2009 APSF Board of Directors' Workshop, a panel of experts in cerebral perfusion concluded that: 1) there is no noninvasive method of defining the safe lower limit of blood pressure for any given patient; 2) blood pressure in the BCP should be adjusted to account for a hydrostatic gradient; 3) deliberate hypotension should be avoided in the $\mathrm{BCP}$; and 4) the maximum reduction in blood pressure from baseline should be $30 \%$ after accounting for a

\footnotetext{
B Lee L, Caplan R. APSF workshop: Cerebral perfusion experts share views on management of head-up cases. APSF Newsletter 2009; 24 : 45-8.
}

hydrostatic gradient in the $\mathrm{BCP} .{ }^{\mathrm{B}}$ On the basis of the limited published data, it appears reasonable to accept these recommendations. However, the data by YaDeau et al. indicate that cerebral oxygenation is well maintained when controlled hypotension is used with regional anesthesia. No obvious adverse effects were noted despite the occurrence of hypotension during $76 \%$ of the observations. ${ }^{6}$ Larger clinical studies are needed to examine the safety of controlled hypotension in the setting of shoulder arthroscopy with regional anesthesia and sedation. In an ongoing investigation, cerebral oximetry is being utilized during shoulder surgery to examine the relationship between blood pressure management and neurocognitive outcomes and sensitive serum biomarkers of neurologic injury (ClinicalTrials.gov number NCT01225185). Until such data are available, it is our view that clinicians should attempt to maintain intraoperative blood pressure as close to baseline values as possible, particularly if general anesthesia is used.

\section{La prise en charge de la tension artérielle pendant une chirurgie de l'épaule en position chaise de plage: que savons-nous?}

L'arthroscopie de l'épaule est l'une des interventions chirurgicales les plus fréquentes en Amérique du Nord. Aux États-Unis, l'anesthésie et la chirurgie d'environ deux tiers de ces patients sont réalisées en position assise ou de chaise de plage. ${ }^{1}$ Bien qu'elle offre de nombreux avantages pour le chirurgien, notamment un risque réduit de traumatisme neurovasculaire, la facilité de conversion vers une approche ouverte et une excellente visualisation intra-articulaire, ${ }^{2}$ la position assise comporte plusieurs défis pour l'anesthésiologiste ainsi que des risques potentiels pour le patient. Des comptes-rendus récents ont décrit des lésions neurologiques catastrophiques chez des patients autrement en bonne santé pendant une chirurgie de l'épaule en position assise. ${ }^{3-5}$ Les auteurs de ces comptes-rendus ont émis l'hypothèse qu'une prise en charge mal adaptée de la tension artérielle a entraîné des réductions de la pression de perfusion cérébrale, ce qui a provoqué une ischémie et une lésion du système nerveux central. À l'heure actuelle, toutefois, plusieurs questions importantes concernant les «meilleures pratiques » pour la prise en charge de la tension artérielle en position assise demeurent sans réponse. Dans ce numéro du Journal, les recherches de YaDeau et coll. ${ }^{6}$ et Trentman et coll. ${ }^{7}$ 
fournissent des données importantes dans ce débat constant quant à la prévalence, aux facteurs de risque et aux conséquences potentielles de l'hypotension pendant une chirurgie de l'épaule en position assise.

Nous ne connaissons pas l'incidence de lésion cérébrale ischémique ou de lésion de la moelle épinière après une chirurgie de l'épaule en position de chaise de plage. En 2003, Bhatti et Enneking ont rapporté le cas d'un patient ayant perdu la vue et manifesté une ophthalmoplégie après une chirurgie de l'épaule. ${ }^{4}$ En 2005, Pohl et Cullen ont décrit quatre cas de lésion cérébrale ou de la moelle épinière graves chez cette population de patients. ${ }^{3}$ Dans un sondage datant de 2009 et interrogeant 287 membres de l'American Shoulder and Elbow Surgeons Society (93 réponses), huit cas de complications vasculaires cérébrales ont été rapportés après une chirurgie de l'épaule, tous chez des patients en position assise. ${ }^{1}$ Une analyse de la base de données des demandes de dédommagement closes (Closed Claims) de l'American Society of Anesthesiologists examinant les lésions de la colonne cervicale nouvellement apparues, publiée cette année, a révélé que $24 \%$ des évènements sont survenus chez des patients subissant une chirurgie en position assise. ${ }^{5}$ Il est probable que l'incidence de ces complications soit considérablement sous-évaluée et rapportée, les chirurgiens et les anesthésiologistes pouvant être réticents à publier des cas dans lesquels des patients en bonne santé ont souffert de lésions neurologiques graves. Les auteurs de cet éditorial ont eu connaissance, via des communications personnelles, de huit cas supplémentaires de lésions au système nerveux central à la suite d'une chirurgie en position assise, lesquels n'ont pas été rapportés dans la littérature. La Fondation américaine pour la sécurité des patients en anesthésie (Anesthesia Patient Safety Foundation-APSF) a maintenant financé un registre national, le NINSS (Neurologic Injury after Non-Supine Shoulder Surgery-http://depts.washington.edu/asaccp/NINS/ index.shtml), afin de déterminer l'incidence des lésions au système nerveux central et les facteurs causaux probables.

Les complications neurologiques rapportées jusqu'à présent étaient la plupart du temps attribuées à une perfusion cérébrale mal adaptée suite à une hypotension peropératoire. D'importants changements hémodynamiques surviennent lorsqu'on bouge le patient d'une position couchée à une position assise. Chez le patient éveillé, la position assise verticale active le système nerveux sympathique, provoquant des augmentations de la résistance vasculaire systémique (30-40\%) et de la tension artérielle systémique (10-15\%) ainsi que des réductions du débit cardiaque $(15-20 \%){ }^{8}$ Sous anesthésie générale, les réactions baroréceptrices sont émoussées, ce qui entraîne une augmentation réduite de la résistance vasculaire systémique, une réduction de la tension artérielle moyenne et une réduction plus grande du débit cardiaque que dans un état éveillé. ${ }^{9}$ Comme on peut s'y attendre, en raison de ces changements physiologiques prononcés, on a observé une incidence élevée d'hypotension peropératoire (12-32\%) chez les patients subissant une chirurgie en position assise sous anesthésie générale. ${ }^{9}$

À l'heure actuelle, deux questions cruciales concernant la prise en charge de la tension artérielle en position assise font l'objet de débats animés. La première touche à la limite inférieure sécuritaire d'autorégulation du cerveau. Traditionnellement, on pensait que la limite inférieure « acceptable » d'autorégulation était une tension artérielle moyenne d'environ $50 \mathrm{mmHg}$. Toutefois, plusieurs études publiées au cours des six dernières décennies ont démontré que ce seuil pourrait être aussi élevé que $60-90 \mathrm{mmHg}$ chez des volontaires normotendus éveillés. ${ }^{10}$ Comme le mentionne un article de synthèse récent, les données probantes à disposition indiquent que la limite inférieure d'autorégulation du cerveau serait une tension artérielle moyenne de $70 \mathrm{mmHg}$ chez les patients non anesthésiés en position couchée. ${ }^{10}$ Ces données indiquent que la pratique, relativement courante, d'une hypotension induite sous anesthésie pourrait mettre en péril la perfusion cérébrale chez certains patients à risque, particulièrement chez les personnes hypertendues dont la limite d'autorégulation inférieure pourrait être déplacée vers la droite. Malheureusement, il n'existe pas de technique non effractive qui permette de déterminer la limite inférieure d'autorégulation chez un patient donné (bien que des données préliminaires suggèrent que l'oxymétrie cérébrale puisse être utile pour définir ce critère d'évaluation). La seconde question importante touche au fait de savoir s'il faudrait corriger la tension artérielle pour tenir compte de la différence de hauteur entre l'emplacement de la mesure (la plupart du temps par un brassard non effractif sur le bras opposé au site de l'opération) et le cerveau. Deux théories sont débattues à l'heure actuelle. Le concept du siphon, ou "modèle fermé », émet l'hypothèse qu'une colonne continue de sang existe entre les membres artériels et veineux de la circulation cérébrale. ${ }^{\mathrm{A}}$ Étant donné que les effets gravitationnels sont identiques dans les membres ascendants et descendants de la boucle, aucun « coefficient de correction » n'est nécessaire; la position assise entraîne des réductions équivalentes de la tension artérielle moyenne et de la pression veineuse centrale, ce qui ne provoque aucun changement au niveau de la pression de perfusion cérébrale. Selon le concept de la cascade, ou «modèle ouvert », des veines qui peuvent s'affaisser empêchent un effet de siphon de fonctionner. ${ }^{\mathrm{A}}$ En raison du phénomène de la gravité, le sang «tombe » du côté veineux du circuit cérébral, et le cœur doit pomper une colonne de sang vers

\footnotetext{
$\overline{\mathrm{A}}$ Kirby RR, Cullen DJ. Lower limit of cerebral autoregulation questioned. APSF Newsletter 2009; 24: 5.
} 
le cerveau. Par conséquent, il faut utiliser un coefficient de correction pour tenir compte du poids de cette colonne de sang (une réduction de la tension artérielle de $1 \mathrm{mmHg}$ pour chaque $1,25 \mathrm{~cm}$ de différence de hauteur entre le site de mesure et le cerveau). À l'heure actuelle, aucun consensus n'existe quant à savoir s'il faut «corriger » ou non la tension artérielle en position assise. En tous les cas, de nombreux (voire la plupart) neuro-anesthésiologistes prennent la tension artérielle en charge selon le concept de la cascade lors d'une chirurgie en position assise et augmentent ainsi le transducteur artériel au niveau du polygone de Willis. Il est toutefois probable que la plupart des anesthésiologistes n'utilise pas un coefficient de correction de la tension artérielle semblable pendant une chirurgie de l'épaule en position assise.

L'étude de YaDeau et coll. a été conçue afin d'examiner l'effet d'une hypotension contrôlée sur l'oxygénation cérébrale chez les patients subissant une arthroscopie de l'épaule en position assise sous anesthésie régionale. ${ }^{6}$ L'oxymétrie cérébrale a été utilisée pour évaluer le bon niveau d'oxygénation du cerveau et, indirectement, celui du débit sanguin cérébral. Bien que les lésions neurologiques soient relativement rares après une chirurgie en position assise, les incidents de désaturation cérébrale majeure - définis en tant qu'une réduction de la saturation cérébrale en oxygène $(\mathrm{rSO} 2) \geq 20 \%$ par rapport aux valeurs de base - sont plus fréquents après une arthroscopie de l'épaule et devraient être considérés comme des «signes d'avertissement précoces » d'une mauvaise perfusion cérébrale, d'ischémie et d'infarctus. ${ }^{11}$ L'étude a été réalisée dans une institution où l'hypotension contrôlée est fréquemment utilisée chez les patients d'orthopédie. L'hypotension a été classée selon des définitions préétablies, et la tension artérielle corrigée afin de tenir compte de la distance entre le cœur et la tête (concept de la cascade). La méthodologie de l'étude comporte de nombreuses limites, par exemple: le type et la profondeur de la sédation n'étaient pas standardisés (le niveau de sédation pourrait avoir une influence sur les valeurs de $\mathrm{rSO} 2$ ); aucun groupe de patients subissant une anesthésie générale n'a été inclus comme cohorte de comparaison; aucune définition normalisée de "l'hypotension contrôlée »n'a été utilisée; plusieurs agents ont été administrés pour induire l'hypotension, lesquels pourraient modifier la vasoréactivité cérébrale et les valeurs de rSO2 par le biais de différents mécanismes; et on a utilisé de l'épinéphrine pour les blocs régionaux, laquelle pourrait avoir un impact sur les valeurs de $\mathrm{rSO} 2$ en augmentant le débit cardiaque et le débit sanguin cérébral pendant les épisodes d'hypotension. Malgré ces limites, l'étude a mis en lumière deux faits importants. En premier lieu, les épisodes de désaturation cérébrale étaient peu fréquents et de courte durée lors de l'utilisation d'une anesthésie régionale. En revanche, $80 \%$ des patients subissent des incidents de désaturation cérébrale majeurs lors de chirurgie en position assise sous anesthésie générale avec ventilation contrôlée. ${ }^{11}$ Ces observations laissent à penser que le débit sanguin cérébral est mieux maintenu en position assise lorsqu'on a recours à une anesthésie régionale. L'incidence réduite d'incidents de désaturation cérébrale chez les patients recevant des blocs des nerfs interscaléniques et supraclaviculaires pourrait être due à une meilleure conservation des indices hémodynamiques sous anesthésie régionale (voir ci-dessus) ou à des valeurs plus élevées de dioxyde de carbone artériel (et aux améliorations associées au niveau du débit sanguin cérébral), lesquelles sont en général observées chez les patients sous sédation respirant spontanément au contraire des patients sous ventilation contrôlée et anesthésie générale. Deuxièmement, aucune association claire n'a été observée entre l'hypotension et les évènements de désaturation cérébrale. La relation entre la tension artérielle et les incidents de désaturation cérébrale n'a pas été examinée lorsque des chirurgies en position assise ont été réalisées sous anesthésie générale. Bien que l'étude en question ici fournisse des données probantes appuyant la sécurité d'une hypotension contrôlée lors de l'utilisation d'une anesthésie régionale, des données supplémentaires sont nécessaires avant de pouvoir recommander une telle pratique. Il est possible qu'une hypotension peropératoire entraîne un dysfonctionnement neurocognitif plus subtil et des lésions cérébrales difficilement dépistées lors d'examens cliniques de routine.

Les incidents de désaturation cérébrale sont fréquents lors de chirurgies en position assise sous anesthésie générale, même lorsque la tension artérielle est maintenue dans une fourchette de $20 \%$ autour des valeurs de base. ${ }^{11}$ L'hypotension est probablement moins bien tolérée chez cette population de patients. Dans l'étude de Trentman et coll., les auteurs ont examiné l'incidence de l'hypotension chez les patients subissant une anesthésie générale pendant une chirurgie de l'épaule en position assise. ${ }^{7}$ Bien qu'il ait été démontré que l'utilisation d'agents antihypertenseurs était associée à une incidence plus élevée d'épisodes d'hypotension chez les patients en position couchée sous anesthésie générale, ${ }^{12}$ cette relation n'a pas été examinée chez les patients en position assise. Les dossiers médicaux ont été rétrospectivement passés en revue afin de déterminer l'incidence d'épisodes d'hypotension (une tension artérielle systolique $\leq 85 \mathrm{mmHg}$ n'a pas été corrigée afin de tenir compte des différences de hauteur entre le site de mesure et le cerveau) chez des patients normotendus ou des patients hypertendus traités. Même après avoir effectué des ajustements afin de tenir compte des facteurs de risque, tels que l'âge et l'indice de masse corporelle, les chercheurs ont observé que l'incidence d'hypotension était significativement supérieure chez les patients recevant des médicaments antihypertenseurs en période préopératoire. 
Bien que sujette aux limites inhérentes aux études rétrospectives (c.-à-d. aucune standardisation en matière d'agent anesthésique, de prise en charge chirurgicale ou de la tension artérielle), cette étude est importante parce qu'elle a démontré que les patients traités avec des agents antihypertenseurs préopératoires courent le risque de manifester de l'hypotension lors d'une anesthésie générale en position assise. Le risque d'hypotension est encore plus élevé si la tension artérielle est mesurée et corrigée selon le concept de la cascade.

Quelle est la gamme de «tensions systémiques cibles idéales » qui peuvent être maintenues en position assise sans causer de lésion neurologique? À l'heure actuelle, les données sont insuffisantes pour répondre à cette question cruciale. Lors de l'Atelier du Conseil d'administration de l'APSF de 2009, un panel d'experts de la perfusion cérébrale ont conclu que: 1) il n'existe pas de méthode non effractive qui permette de définir la limite inférieure sécuritaire de la tension artérielle chez un patient donné; 2) la tension artérielle en position assise devrait être ajustée pour tenir compte d'un gradient hydrostatique; 3) il faut éviter une hypotension délibérée en position assise; et 4) la réduction maximale de la tension artérielle par rapport aux valeurs de base devrait être de $30 \%$ après avoir pris en considération un gradient hydrostatique en position assise. ${ }^{\mathrm{B}}$ En se fondant sur les données publiées limitées, il semble raisonnable d'accepter ces recommandations. Toutefois, les données de YaDeau et coll. indiquent que l'oxygénation cérébrale est bien maintenue lorsqu'on a recours à une hypotension contrôlée avec une anesthésie régionale. Aucun effet secondaire néfaste évident n'a été noté malgré la survenue d'épisodes d'hypotension pendant $76 \%$ des cas observés. ${ }^{6}$ Des études cliniques d'envergure plus importante sont nécessaires afin d'étudier la sécurité de l'hypotension contrôlée dans le cadre d'une arthroscopie de l'épaule faite sous anesthésie régionale et sédation. Dans une étude en cours, l'oxymétrie cérébrale est utilisée pendant une chirurgie de l'épaule afin d'examiner la relation entre la prise en charge de la tension artérielle et les devenirs neurologiques ainsi que les biomarqueurs sériques sensibles à une lésion neurologique (numéro ClinicalTrials.gov NCT01225185). En attendant que de telles données soient disponibles, les cliniciens devraient, selon nous, tenter de maintenir la tension artérielle peropératoire aussi proche des valeurs de base que possible, tout particulièrement en cas d'anesthésie générale.

Funding source Department of Anesthesiology, NorthShore University HealthSystem.

Conflicts of interest Dr. Glenn Murphy has received consulting fees from CASMED.

\section{References}

1. Friedman DJ, Parnes NZ, Zimmer Z, Higgins LD, Warner JJ. Prevalence of cerebrovascular events during shoulder surgery and association with patient position. Orthopedics 2009; 32: 256.

2. Skyhar MJ, Altchek DW, Warren RF, Wickiewicz TL, O'Brien SJ. Shoulder arthroscopy with the patient in the beach-chair position. Arthroscopy 1988; 4: 256-9.

3. Pohl A, Cullen DJ. Cerebral ischemia during shoulder surgery in the upright position: a case series. J Clin Anesth 2005; 17: 463-9.

4. Bhatti MT, Enneking FK. Visual loss and ophthalmoplegia after shoulder surgery. Anesth Analg 2003; 96: 899-902.

5. Hindman BJ, Palecek JP, Posner KL, et al. Cervical spinal cord, root, and bony spine injuries: a closed claims analysis. Anesthesiology 2011; 114: 782-95.

6. YaDeau JT, Liu SS, Bang H, et al. Cerebral oximetry desaturation during shoulder surgery performed in a sitting position under regional anesthesia. Can J Anesth 2011; 58. DOI:10.1007/ s12630-011-9574-7.

7. Trentman TL, Fassett SL, Thomas JK, et al. More hypotension in patients taking antihypertensives preoperatively during shoulder surgery in the beach chair position. Can J Anesth 2011; 58. DOI: 10.1007/s12630-011-9575-6.

8. Smith JJ, Porth CM, Erickson M. Hemodynamic response to the upright posture. J Clin Pharmacol 1994; 34: 375-86.

9. Porter JM, Pidgeon C, Cunningham AJ. The sitting position in neurosurgery: a critical appraisal. Br J Anaesth 1999; 82: 117-28.

10. Drummond JC. The lower limit of autoregulation: time to revise our thinking? Anesthesiology 1997; 86: 1431-3.

11. Murphy GS, Szokol JW, Marymont JH, et al. Cerebral oxygen desaturation events assessed by near-infrared spectroscopy during shoulder arthroscopy in the beach chair and lateral decubitus positions. Anesth Analg 2010; 111: 496-505.

12. Colson P, Saussine M, Seguin JR, Cuchet D, Chaptal PA, Roquefeuil B. Hemodynamic effects of anesthesia in patients chronically treated with angiotensin-converting enzyme inhibitors. Anesth Analg 1992; 74: 805-8.

\footnotetext{
${ }^{\mathrm{B}}$ Lee L, Caplan R. APSF workshop: Cerebral perfusion experts share views on management of head-up cases. APSF Newsletter 2009; 24: 45-8.
} 